Service social

\title{
Réflexion critique sur l'approche milieu de vie dans les centres d'hébergement et de soins de longue durée : un éclairage nouveau à partir de la promotion de la santé
}

\author{
Jacky Ndjepel
}

Volume 60, numéro 2, 2014

URI : https://id.erudit.org/iderudit/1027993ar

DOI : https://doi.org/10.7202/1027993ar

Aller au sommaire du numéro

Éditeur(s)

École de service social de l’Université Laval

ISSN

1708-1734 (numérique)

Découvrir la revue

Citer cet article

Ndjepel, J. (2014). Réflexion critique sur l'approche milieu de vie dans les centres d'hébergement et de soins de longue durée : un éclairage nouveau à partir de la promotion de la santé. Service social, 60(2), 89-100.

https://doi.org/10.7202/1027993ar
Résumé de l'article

Depuis quelques années, l'approche milieu de vie (AMV) est devenue populaire parce qu'elle représente une avenue intéressante pour améliorer la qualité de vie des résidants dans les milieux d'hébergement. Malgré sa pertinence, l'AMV peine encore à être intégrée dans la pratique quotidienne des centres d'hébergement. En 2014, seuls quelques-uns se distinguent à cet égard. De nombreux facteurs expliquent cette situation notamment les difficultés que rencontrent les professionnels dans la réalisation de leurs tâches, le manque de personnel, une plus importante charge de travail et surtout l'assise conceptuelle de l'AMV qui semble encore mal comprise par plusieurs. Au plan organisationnel, malgré la volonté politique de transformer les centres en milieux de vie, ceux-ci fonctionnent encore avec des logiques institutionnelles centrées sur l'offre de soins. Afin de pallier ces difficultés, certains développements conceptuels récents en promotion de la santé, en l'occurrence le modèle de l'AMV de Poland et Dooris (2010), pourraient être mis à contribution afin d'améliorer l'implantation de l'AMV dans les CHSLD. 


\title{
Réflexion critique sur l'approche milieu de vie dans les centres d'hébergement et de soins de longue durée : un éclairage nouveau à partir de la promotion de la santé
}

\author{
NDJEPEL, Jacky \\ Doctorante en santé communautaire \\ Faculté des sciences infirmières \\ Université Laval
}

\section{RÉSUMÉ}

Depuis quelques années, l'approche milieu de vie (AMV) est devenue populaire parce qu'elle représente une avenue intéressante pour améliorer la qualité de vie des résidants dans les milieux d'hébergement. Malgré sa pertinence, l'AMV peine encore à être intégrée dans la pratique quotidienne des centres d'hébergement. En 2014, seuls quelques-uns se distinguent à cet égard. De nombreux facteurs expliquent cette situation notamment les difficultés que rencontrent les professionnels dans la réalisation de leurs tâches, le manque de personnel, une plus importante charge de travail et surtout l'assise conceptuelle de l'AMV qui semble encore mal comprise par plusieurs. Au plan organisationnel, malgré la volonté politique de transformer les centres en milieux de vie, ceux-ci fonctionnent encore avec des logiques institutionnelles centrées sur l'offre de soins. Afin de pallier ces difficultés, certains développements conceptuels récents en promotion de la santé, en l'occurrence le modèle de l'AMV de Poland et Dooris (2010), pourraient être mis à contribution afin d'améliorer l'implantation de l'AMV dans les CHSLD.

Mots clés : Approche milieu de vie, Promotion de la santé, CHSLD, Modèle écologique.

\begin{abstract}
ABSTACT
In the few past years, the approach "milieu de vie" (AMV) has gained popularity since it represents an interesting avenue to improve the quality of life of people living in long-term care homes. Despite its relevancy, the AMV is still not fully integrated in the daily practice of nursing homes. In 2014, only a handful of these homes differed from the others, regarding this approach. Many factors can explain this situation such as the obstacles that health professionals face when carrying out their tasks, staff shortage, a greater workload and the conceptual basis of the AMV which does not seem to be fully understood by many people. At the organizational level, despite a political will to transform long-term care homes, they are still run with a more institutional reasoning centered on offering care. To overcome these difficulties, certain recent conceptual developments in health promotion, such as the model of AMV of Poland \& Dooris (2010), could be used to improve the AMV in long-term care homes.
\end{abstract}

Key words: Setting approach or approach "milieu de vie"; Health promotion; Long-term care homes; Ecological model. 


\section{INTRODUCTION}

Dans les années 2000, en vue de contrer les effets délétères de l'institutionnalisation et d'assurer aux personnes hébergées en centres d'hébergement et de soins de longue durée (CHSLD), des services adaptés à leurs besoins, le ministère de la Santé et des Services sociaux du Québec (MSSS) enclenche une réflexion sur la transformation des milieux de soins en milieux de vie. En 2003, cette volonté se matérialise par la publication des orientations ministérielles intitulées Un milieu de vie de qualité pour les personnes hébergées en CHSLD (MSSS, 2003). Cette nouvelle démarche, appelée « approche milieu de vie » (AMV), avait pour principal objectif d'améliorer les soins et les services dans les CHSLD et de garantir aux personnes hébergées une meilleure qualité de vie et de bien-être. L'AMV devait ainsi contribuer à modifier les pratiques professionnelles axées exclusivement sur le soin pour celles qui tiennent compte des besoins et des choix exprimés par les résidants (Fahey, 2003 ; Aubry, Godin et Couturier, 2011 ; Aubry, Couturier et Gilbert, 2013).

Malgré sa pertinence, l'AMV peine encore à s'implanter dans les CHSLD à l'heure actuelle (Aubry, Couturier et Gilbert, 2013). De 2004 à 2013, le MSSS a effectué des visites d'appréciation dans différentes régions du Québec en vue d'évaluer sa mise en œuvre. Plusieurs rapports d'évaluation soulignent que quelques structures d'accueil se distinguent notamment sur le plan des soins de base et du respect de l'identité des résidants. Cependant, des lacunes ont été observées sur la formation reçue par les intervenants (Aubry, Godin et Couturier, 2011 ; Aubry, Couturier et Gilbert, 2013). Des recommandations ont été formulées afin que les centres qui n'appliquent pas encore l'AMV puissent devenir des milieux de vie avec l'appui de plans d'amélioration mis à leur disposition (MSSS, 2013).

Plusieurs auteurs ont identifié une diversité de facteurs à différents niveaux pour expliquer l'application difficile de l'AMV dans les CHSLD (Alderson, 2006 ; Aubry, Godin et Couturier, 2011 ; Soulières et Ouellette, 2012 ; Aubry, Couturier et Gilbert, 2013). Selon nous, l'AMV promue par le MSSS comporte quelques manquements sur le plan conceptuel qui méritent qu'on les aborde. Ces manquements constituent un autre frein à son implantation. Cet article voudrait donc y revenir en posant un regard critique sur l'AMV. En s'inspirant des développements conceptuels récents en promotion de la santé (PS), et en considérant notamment le modèle de l'AMV de Poland et Dooris (2010), des éléments nouveaux permettront de mieux comprendre l'AMV et d'améliorer ainsi son implantation en CHSLD. Nous présenterons tout d'abord l'AMV telle que promue par le MSSS, nous aborderons ensuite les principaux problèmes qui rendent son application difficile. La conceptualisation de l'AMV en PS fera l'objet de la dernière articulation du texte. Un accent particulier sera mis sur l'AMV de Poland et Dooris (2010).

\section{L'AMV telle que promue par le MSSS}

Selon le MSSS (2003; 2004), l'AMV consiste à transformer les CHSLD en les invitant à concevoir leurs services non plus à partir des besoins organisationnels et professionnels, mais bien à partir des besoins des personnes hébergées. Ainsi, les CHSLD seraient désormais considérés comme un nouveau «chez-soi » des résidants, c'est-à-dire qu'ils devraient leur 
permettre de retrouver dans les milieux d'hébergement une forme de vie adaptée à leurs valeurs et ressemblant à leur environnement habituel. Afin de guider la mise en œuvre de l'AMV, le MSSS (2003) a publié des orientations précises à ce sujet. Celles-ci se regroupaient en deux principales composantes: une composante humaine et une composante organisationnelle (Aubry, Godin et Couturier, 2011).

Sur le plan humain, l'AMV est centrée essentiellement sur les résidants. La démarche se veut à la fois globale, adaptée, positive, personnalisée, participative et interdisciplinaire, et s'appuie sur des valeurs comme le respect de la personne hébergée, son intégrité, sa dignité et la valorisation de ses droits et libertés (MSSS, 2003). De façon concrète, cette pratique se traduit par l'humanisation des interventions, une réponse adéquate aux besoins et attentes des personnes hébergées et un accompagnement qui favorise leur autonomie et l'implication de leurs proches. Pour y parvenir, les intervenants doivent faire montre d'ouverture et de flexibilité dans la réalisation de leurs tâches ; être à l'écoute des résidants et de leur famille ; respecter leurs choix et leurs décisions dans l'accompagnement. Au plan organisationnel, des efforts devaient être faits afin que l'environnement physique puisse se rapprocher autant que possible du milieu naturel du résidant. Les CHSLD devenaient ainsi des lieux accueillants, chaleureux et personnalisés. Outre la modification physique des lieux, l'AMV suppose de favoriser une relation de proximité entre les résidants et les intervenants d'une part et une souplesse au niveau des horaires d'autre part. Aussi pour y parvenir, le MSSS (2003) proposait qu'une formation soit donnée aux intervenants pour améliorer leur communication et préciser leurs rôles et responsabilités pour la mise en œuvre de l'AMV.

\section{Les difficultés liées à l'implantation de l'AMV}

Même si l'AMV repose sur différents modèles théoriques et propose plusieurs techniques d'intervention, elle ne semble pas encore pas bien comprise par divers acteurs. Certains professionnels en ont une compréhension plus ou moins floue (Aubry, Godin et Couturier, 2011). D'autres auteurs remarquent que c'est un «terme galvaudé qui prend une signification bien différente selon l'interlocuteur » (Soulières et Ouellette, 2012, p. 27). Le manque de clarté et les diverses interprétations de l'AMV créent une confusion et en suscitent une vision plurielle. Dans son ouvrage Gérontologie appliquée, Laforest a écrit : "l'approche milieu de vie est plus large que le concept de milieu de soins : il l'englobe, comme il englobe les autres aspects de la vie » (Laforest, 1997, p. 78). On retrouve dans l'AMV un éventail de finalités, d'orientations et de pratiques plus ou moins compatibles (Amyot, 2008). L'absence de définition explicite de l'AMV conduit à une compréhension diversifiée de l'approche. Cette situation crée des « flous » chez les divers acteurs et mène à des pratiques différentes et parfois contradictoires. À l'exception des orientations ministérielles, à notre connaissance, il ne semble pas exister un cadre de référence qui puisse permettre de mieux expliciter cette approche et ses concepts clés, par exemple ce qu'on entend par milieu de vie.

Sur le plan des pratiques, les professionnels en général et, particulièrement, les préposés aux bénéficiaires (PAB) relèvent les difficultés rencontrées dans la mise en place de l'AMV. À ce sujet, les $P A B$ relèvent en premier lieu une certaine inadéquation entre la formation qu'ils reçoivent et leur pratique sur le terrain (Aubry, Godin et Couturier, 2011). En effet, aucun module 
abordant l'AMV ne faisait partie du contenu de leur formation. Deuxièmement, les difficultés organisationnelles rencontrées par les intervenants dans leur pratique quotidienne n'ont pas été prises en compte dans la formation (Aubry, Godin et Couturier, 2011). En outre, l'insuffisance de personnel pour combler tous les besoins, le manque de temps et les conditions de travail parfois difficiles freinent la mise en place de l'AMV (Aubry, Godin et Couturier, 2011 ; Aubry, Couturier et Gilbert, 2013) et contribuent très souvent à fragiliser la santé physique et psychologique du personnel (Cloutier, David et Teiger, 2003).

Quant aux infirmières, elles semblent avoir perdu leur statut et estiment que les familles occupent plus de place et sont devenues leurs «boss» (Alderson, 2006). Cette auteure remarque par ailleurs que la décision ministérielle de mettre en place l'AMV a été trop brusque et n'a pas été négociée avec les intervenants. Ni leurs besoins ni leurs points de vue n'ont été pris en compte. La seule fois que les orientations ministérielles évoquaient les intervenants, le MSSS les invitait à plus d'ouverture et de flexibilité dans la réalisation de leurs tâches (Alderson, 2006). Pourtant, l'introduction d'un changement est « une démarche qui se déroule à partir d'une réelle réflexion avec l'ensemble des acteurs pour comprendre les enjeux de chacun » (Eynard, Piou et Villez, 2009, p. 27). Le modèle de Poland et Dooris (2010) peut être utile pour réfléchir et guider l'implantation de l'AMV dans les CHSLD puisqu'il sollicite la participation de tous les acteurs dans la mise en place d'un milieu de vie.

Selon les PAB et les infirmières, l'AMV se réduit essentiellement à satisfaire les besoins du résidant et à favoriser une relation entre la personne hébergée et ses proches (Ouellette, 2012 ; Soulières et Ouellette, 2012). Les attentes du personnel ou les difficultés qu'il rencontre dans la mise en œuvre de l'AMV ne semblaient pas être une priorité pour le MSSS. Or, parler d'une AMV selon les approches théoriques récentes en PS, c'est précisément tenir compte à la fois des personnes qui y vivent et qui y travaillent et des relations qu'elles entretiennent. Bref, c'est s'intéresser à toutes les personnes qui se rencontrent dans ce milieu (résidants, professionnels, visiteurs, administrateurs, etc.) et aux dynamiques qui s'y construisent.

Dans le glossaire de PS de l'Organisation mondiale de la santé (OMS), un milieu de vie favorable à la santé représente «le lieu ou le contexte social dans lequel les individus s'investissent dans leurs activités quotidiennes et dans lequel les facteurs personnels, organisationnels et environnementaux influent sur leur santé et leur bien-être » (Nutbeam, 1999, p. 19). En d'autres termes, mettre en place une AMV consiste à créer un environnement favorable pour tous (les résidants, les travailleurs, etc.). II ne s'agit pas seulement de considérer les intervenants comme des professionnels qui doivent s'ouvrir à une nouvelle approche de travail, mais plutôt comme des cibles spécifiques avec lesquelles il faudrait composer et adapter une stratégie appropriée à leur situation et évidemment viser leur bien-être. Si une nouvelle pratique ne correspond pas au milieu ou n'y est pas acceptée, elle ne s'enracinera pas (Poland et Dooris, 2010). Pour qu'elle soit une réussite, l'AMV doit tenir compte du contexte sans perdre de vue toutes les personnes qui s'y trouvent (Poland et Dooris, 2010). Les changements s'opèrent par des personnes qui, exposées à des programmes ou des interventions qui tiennent compte de leurs besoins et attentes, s'impliquent et décident de changer les choses (Pawson et Tilley, 1997). La participation, l'autonomisation, l'équité, le partenariat, la durabilité sont des valeurs qui caractérisent une AMV dans une perspective de PS (Poland et Dooris, 2010). Ces 
valeurs centrales ne transparaissent pas dans l'AMV promue par le MSSS. Les intégrer dans le processus d'implantation augurera probablement de meilleurs résultats. De nombreuses expériences existent maintenant avec l'AMV. Les plus importantes sont les villes/communes, les écoles, les hôpitaux, les entreprises. Ces expériences fructueuses montrent que l'implantation d'une AMV, même si elle soulève des défis, peut se faire.

Au plan organisationnel, le modèle biomédical est encore présent dans les CHSLD (nombre de soins requis, de lits, chambres identifiées par un numéro et non par le nom de la personne). Les CHSLD fonctionnent surtout avec des logiques institutionnelles centrées sur l'offre de soins (Charpentier, Guberman et Soulières, 2010) plutôt qu'axées sur une AMV. D'après Villez (2007), le projet de soins prime sur le projet de vie dans ces milieux et la vie quotidienne s'articule surtout autour de la satisfaction des besoins primaires et de la distribution des soins. Même les activités socioculturelles y ont une visée thérapeutique. En raison de son approche non médicale, l'AMV est souvent moins comprise par les responsables et les professionnels qui s'inscrivent dans une perspective biomédicale de la santé (Kickbusch, 1996). Ce constat montre que l'AMV invite à une nouvelle vision de la santé. Une santé qui ne s'enracine pas seulement dans le soin mais qui va au-delà de cette dimension. Afin de mieux comprendre ce qu'est une $A M V$, il faudrait s'inscrire dans un nouveau paradigme, une nouvelle conception de la santé qui est développée depuis plusieurs années en PS.

\section{La notion de milieu de vie en promotion de la santé}

Avant d'aborder la notion de milieu de vie en PS, il est pertinent de revenir brièvement sur ce qu'est la PS. La définir n'est pas une tâche aisée, mais on peut y répondre à partir de son émergence en tant que champ de pratique et de recherche, sa conception positive de la santé et les valeurs qu'elle préconise.

\subsection{Qu'est-ce que la promotion de la santé (PS) ?}

Dans les années 1980, l'émergence de la PS marque une volonté manifeste de créer une nouvelle conception de la santé. Longtemps influencé exclusivement par le modèle biomédical, le domaine de la santé s'est orienté vers un nouveau cadre de référence pouvant rendre compte de façon plus pertinente de l'état de santé des populations. II s'agissait ainsi de se soustraire de la vision traditionnelle de la santé qui ne privilégiait que les soins pour adopter une vision plus globale (Lalonde, 1974). Ce regard nouveau suggérait, d'une part, de penser la santé à travers d'autres facteurs: l'environnement physique et social, les habitudes de vie, l'organisation des soins de santé (O’Neill, Pederson, Dupéré et Rootman, 2006) et, d'autre part, d'accorder de l'importance aux interventions qui préviennent les maladies et promeuvent le bien-être des populations, plutôt que des actions correctives pour traiter des conséquences (Houéto et Laverack, 2014). De cette innovation, la conception biomédicale de la santé a progressivement glissé vers une approche socio-environnementale appelée «promotion de la santé ». Celle-ci va s'intéresser aux déterminants de la santé et valoriser le travail intersectoriel par la participation de tous les acteurs concernés (spécialistes, professionnels, administrateurs, communautés).

La charte d'Ottawa, document référentiel de la PS, est adoptée au Canada lors de la première conférence internationale sur la PS en 1986. Cette rencontre a permis d'identifier de 
nouvelles stratégies d'intervention pour améliorer la santé des populations dans une perspective de PS. Cinq niveaux d'action ont ainsi été définis:1) l'élaboration de politiques publiques favorables à la santé ; 2) la création d'environnements favorables à la santé ; 3) le renforcement de l'action communautaire ; 4) le développement des aptitudes individuelles et 5) la réorientation des services de santé (OMS, 1986). D'après l'OMS, la PS est le processus qui confère aux populations les moyens d'assurer un plus grand contrôle sur leur propre santé et de l'améliorer (OMS, 1986). C'est une démarche qui est axée sur des personnes, leur situation, leurs besoins, leur environnement physique, psychologique et social, plutôt que sur un problème médical. La charte d'Ottawa peut être considérée comme un catalyseur de l'AMV (Dooris, 2006). La charte a effectivement mis en évidence l'incidence des milieux de vie sur la santé des personnes et l'importance de prendre en compte les facteurs socio-environnementaux dans la compréhension des problèmes de santé.

\subsection{La conceptualisation de l'AMV en promotion de la santé}

Avec l'avènement de la PS, la question des milieux de vie favorables à la santé est devenue un élément important et une composante essentielle dans les stratégies pour améliorer la santé des populations (O'Neill et al., 2012). Depuis plusieurs années, le concept « milieux favorables à la santé » a été développé par différents auteurs (Green, Rootman et Poland, 1999 ; Dooris et al., 2007 ; Poland et Dooris, 2010). Le milieu de vie se rapporte aux aspects physiques et sociaux de l'environnement dans lequel nous vivons. II constitue par ailleurs le lieu où des individus se rencontrent et façonnent leur environnement pour y créer un espace de bien-être et de santé (OMS, 1998). Comme milieux de vie, on compte des écoles, des milieux de travail, des milieux virtuels, des villes, des hôpitaux, des centres d'hébergement, etc.

Différents auteurs ont précisé les caractéristiques d'une AMV en PS (Poland, Green et Rootman, 2000 ; Dooris, 2006 ; O'Neill et al., 2012). Parmi les principales, on retient qu'une AMV suggère des approches globales qui se veulent écologiques. Les interventions visent ainsi des changements au niveau des individus, des organisations et des environnements dans lesquels l'AMV prend place. Mentionnons également que l'AMV se met en place selon la réalité de chaque milieu et en privilégiant autant que possible l'implication de toutes les parties prenantes. De par ces caractéristiques et en se référant à la définition de l'OMS (Nutbeam, 1999), I'AMV fait partie des approches écologiques. Parce que l'AMV s'inscrit dans une perspective écologique et systémique, elle touche à plusieurs cibles et niveaux d'intervention, par opposition à une seule telle que véhiculée par l'AMV du MSSS.

L'existence de différents milieux de vie (les uns plus petits que les autres ou encore faisant partie de milieux plus grands) a amené la PS à développer une compréhension commune de l'AMV. Ce qui permettait de mieux orienter les pratiques sur le terrain (Dooris, 2006). Bon nombre d'auteurs ont ainsi proposé des cadres permettant de travailler dans une AMV. D'après O'Neill et ses collègues (2012), la notion de milieu de vie favorable à la santé s'inspire d'une approche holistique et écologique. En raison de la complexité des milieux de vie, les modèles écologiques ont souvent été utilisés dans les interventions visant les milieux de vie. Ces modèles permettent de collaborer avec différentes cibles à la fois et à divers niveaux. À ce sujet, Stokols (1996) souligne qu'un programme écologique inclut des actions visant plusieurs cibles 
(individus et différentes facettes de l'environnement), et ce, dans plusieurs sites d'intervention. Les milieux et les cibles constituent ainsi les dimensions clés de l'intégration de l'approche écologique dans les programmes de PS (Richard, Gauvin et Raine, 2011). L'AMV telle que promue par le MSSS ne s'en inspire pas. Pourtant, l'intégration de ces dimensions la bonifierait grandement et faciliterait par conséquent son implantation. Ces composantes clés se retrouvent dans le modèle théorique de l'AMV de Poland et Dooris (2010) et peuvent contribuer à améliorer l'application de l'AMV en CHSLD.

\subsection{L'approche conceptuelle de l'AMV de Poland et Dooris (2010)}

C'est un modèle d'inspiration écologique et systémique, qui met en exergue les conditions pour qu'un milieu de vie se développe et accède à un changement d'un point de vue global. Les valeurs, les méthodes et les approches que ce modèle préconise sont pertinentes pour la mise en œuvre de l'AMV dans la pratique de tous les jours. Selon Poland et Dooris (2010), l'AMV se caractérise par trois dimensions.

-Une dimension socioécologique qui postule que la santé est déterminée par l'interaction des facteurs environnementaux, organisationnels et personnels. Pour implanter une AMV, on doit prendre en considération ces différents facteurs et niveaux d'intervention et s'assurer de la participation de tous les acteurs clés en tentant évidemment de modifier les structures de pouvoir. De fait, cette dimension suppose d'adopter une démarche holistique en ayant en vue tous les acteurs, les niveaux d'interventions et en identifiant les stratégies adéquates par groupe cible et palier d'intervention. Cette démarche suggère par ailleurs d'allier les approches salutogénique (salutogenèse) et pathogénique (pathogenèse). Ainsi, les interventions ne seront pas exclusivement centrées sur le soin (traitement des maladies) mais devront s'inscrire dans une perspective positive de création de la santé. II s'agira ainsi de valoriser le plus possible les atouts des divers acteurs plutôt que leurs déficits pour créer un milieu de vie favorable à la santé pour tous (résidants, professionnels, etc.).

-Une dimension systémique qui souligne la nécessité de considérer la complexité des différents environnements tant physiques que sociaux où l'AMV est implantée et des dynamiques d'influence qui s'y développent. De fait, chaque milieu de vie s'inscrit dans un tout plus grand, un « système ouvert » qui échange avec d'autres environnements. L'approche systémique permet alors d'analyser tous les éléments du système dans sa globalité, de cerner les liens, les relations et les interactions entre les éléments constitutifs du système et de mieux comprendre quelles sont les interventions qui marchent, pour qui et dans quelles conditions (Bériot, 2006). Adopter une perspective systémique dans la mise en œuvre de l'AMV facilite premièrement le repérage des lieux et des acteurs clés pour l'intervention (résidants, intervenants, administrateurs, etc.). Deuxièmement, cette perspective fournit des méthodes adaptées pour conduire les changements souhaités, en l'occurrence l'élaboration et la mise en œuvre des politiques favorables à la santé, l'éducation par les pairs, le marketing social, la modification des environnements (Poland et Dooris, 2010). En évitant des représentations linéaires de causalité, l'approche systémique contribue enfin à identifier les dynamiques d'influence qui traversent l'organisation où l'AMV veut s'implanter. 
- La dernière dimension se traduit par le développement et le changement du milieu dans sa globalité. Selon Dooris (2009), toute nouvelle initiative comporte des défis. Le plus important consiste à arriver à un changement où tout le monde s'entend pour dire que l'intervention est pertinente et que la PS est l'affaire de tous. Cette dimension rappelle donc l'importance de ne pas concentrer les efforts sur un seul élément du système pour mettre en place une AMV. Cette action est peu susceptible de provoquer le changement nécessaire et les résultats attendus. Cette dimension propose d'agir plutôt de façon globale, sur l'ensemble des facteurs et des cibles identifiés à l'aide de multiples stratégies, chaque stratégie orientant ainsi les activités à réaliser pour implanter l'approche. Derrière chacune des stratégies, il faut nécessairement penser aux différentes parties prenantes pour l'implantation de l'AMV, en l'occurrence les instances, les décideurs, les professionnels, qui peuvent contribuer à améliorer son application. Ce niveau consiste donc à introduire et à gérer le changement dans le système à tous les paliers de l'intervention (individu, organisation, etc.).
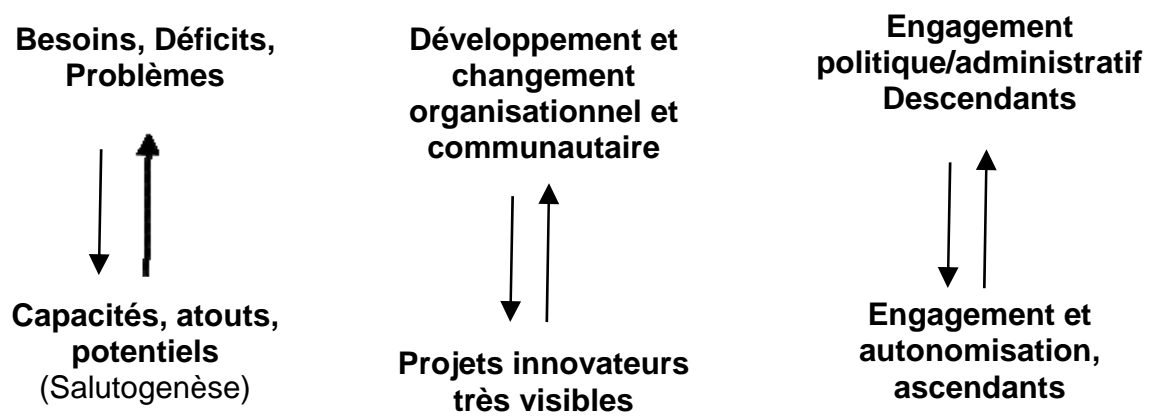

Programmes institutionnels et activités centrales

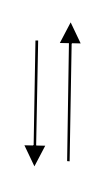

Programme de la santé publique

Figure 1 : Modèle conceptuel de l'AMV de Poland et Dooris (2010).

Ex. : Élaboration/mise en œuvre des politiques, modification de l'environnement,
marketing social, éducation par les pairs, étude d'impact
marketing social, éducation par les pairs, étude d'impact

\section{Valeurs}

Participation, autonomisation, équité, partenariat, durabilité

Pour mettre en place une AMV, ce modèle mise sur l'équilibre entre toutes ses composantes, tout en considérant les trois dimensions évoquées. L'accent ne peut être mis sur une seule composante au détriment d'une autre. Poland et Dooris proposent en premier lieu de capitaliser sur les forces, succès et atouts, plutôt que de se concentrer sur les faiblesses des acteurs clés. L'AMV du MSSS pourrait par exemple être bonifiée en valorisant le travail qu'accomplissent les $P A B$, les infirmières auprès des résidants. Souligner leur contribution importante dans l'accompagnement des résidants, leur donner la parole afin que ces derniers se sentent écoutés, et tenir compte de leurs besoins et attentes. En deuxième lieu, Poland et Dooris proposent d'initier des projets innovateurs et visibles afin de stimuler le développement et le changement dans les pratiques tant au niveau individuel qu'organisationnel, voire communautaire. À ce sujet, le MSSS aurait pu tout d'abord lancer un projet pilote afin 
d'expérimenter l'AMV dans un CHSLD. Les résultats obtenus à l'issue du projet ainsi que les connaissances acquises auraient contribué à déterminer les stratégies à utiliser pour chacune des cibles et comment travailler avec elles pour réussir à implanter l'AMV. En troisième lieu, Poland et Dooris demandent d'allier les visions descendante (top down) et ascendante (bottom up) dans la mise en place d'une AMV, tout en reconnaissant les valeurs de chacune d'elles et en favorisant un leadership à la fois « politique » et «technique ». Un dialogue est nécessaire entre toutes les personnes qui tentent de créer une AMV, ainsi que la prise en compte des processus ascendants et descendants. Quant à la dernière composante du modèle, elle invite à véhiculer le langage de la PS dans le milieu de vie que nous voulons créer. II s'agit de montrer que la mise en place de l'AMV peut apporter une différence significative dans l'amélioration de la santé des personnes hébergées, mais aussi des professionnels qui travaillent auprès d'elles. Toutes les actions proposées par Poland et Dooris pour l'implantation d'une AMV sont encadrées par des valeurs : la participation, l'autonomisation, l'équité, le partenariat, durabilité.

Ce modèle, comme la plupart des approches écologiques, a ses limites. En raison de la complexité d'une part des milieux dans lesquels on veut implanter une AMV et d'autre part le grand nombre cible à intégrer dans cette démarche, atteindre l'équilibre recherché par Poland et Dooris (2010) demeure laborieux. De fait, la participation active des différentes cibles passe par une modification des structures de pouvoir. Ce qui peut entraîner des tensions et freiner la mise en œuvre de l'AMV. En outre, évaluer ce type de modèle afin de mesurer son impact représente une tâche ardue. Aucune composante du modèle ne met en évidence son évaluation. Si une composante évaluative y était ajoutée, celle-ci permettrait de mesurer la mise en place de l'AMV à partir de ce modèle. Les indicateurs et les éléments à évaluer seraient mieux explicités. Malgré ces limites, les modèles écologiques, et particulièrement celui de Poland et Dooris, apportent un éclairage nouveau de l'AMV. Tenir compte de cette lecture peut améliorer la compréhension de ce qu'est une AMV dans une perspective de PS et évidemment peut aider à surmonter les défis énoncés.

\section{CONCLUSION}

« Faire d'un centre d'accueil un milieu de vie est un objectif qui ne peut être réservé à quelques membres du personnel de l'institution, spécialistes des sciences humaines ou des relations interpersonnelles » (Laforest, 1997, p. 80). En effet, implanter une AMV ne consiste pas uniquement à considérer les besoins des résidants, mais nécessite d'adopter une démarche globale et complexe, en valorisant la participation des acteurs clés. La transformation des milieux de soins en milieux de vie passe donc par l'implication de tous les acteurs clés, tant au niveau opérationnel que global. L'établissement d'un dialogue et une compréhension partagée par tous les acteurs des enjeux liés à l'application de l'AMV permettra de trouver des solutions pour y répondre de manière concertée. Le plus important réside dans la mise en avant des valeurs véhiculées par l'AMV. Les promouvoir produit de meilleurs résultats (Poland et Dooris, 2010). On pourrait également passer par un croisement de savoirs où des universitaires, des agents du MSSS, les intervenants (infirmières, préposés aux bénéficiaires, etc.) et les résidants réfléchiraient ensemble afin de déterminer les modalités qui conviennent pour bonifier la mise en œuvre de l'AMV dans les CHSLD. 


\section{REMERCIEMENTS}

L'auteure souhaite remercier Sophie Dupéré et Nicolas Vonarx, professeurs à l'université Laval à la Faculté des sciences infirmières, pour leurs précieux commentaires qui ont permis de bonifier le contenu présenté.

NDJEPEL, Jacky

Doctorante en santé communautaire

Faculté des sciences infirmières

Université Laval

\section{BIBLIOGRAPHIE}

Alderson, M. (2006). "Milieu de vie" ou "milieu de soins": un débat chaud en centres d'hébergement et de soins de longue durée », Vie et vieillissement, vol. 5, n 1, p. 9-15.

Amyot, J.-J. (2008). Travailler auprès des personnes âgées, Paris, Dunod.

Aubry, F., K. Godin et Y. Couturier(2011). « Les préposées aux bénéficiaires face au processus d'implantation de l'approche milieu de vie en soins de longue durée: critiques et perspectives d'avenir », Vie et vieillissement, vol. 9, $\mathrm{n}^{0} 3$, p.36-43.

Aubry, F., Y. Couturier et F. Gilbert (2013). «L'application de l'approche milieu de vie en établissement d'hébergement de soins de longue durée par les préposés aux bénéficiaires du point de vue des préposés, infirmières auxiliaires et infirmières », L'infirmière clinicienne, vol. $10, n^{0} 1, p .52-61$.

Bériot, D. (2006). Manager par l'approche systémique, Paris, Éditions d’Organisation.

Caillard, J-F. (2012). «La promotion de la santé des travailleurs tout au long de la vie professionnelle », Archives des Maladies Professionnelles et de l'Environnement, vol. 73, $\mathrm{n}^{0} 3$, p. 276-279.

Charpentier, M., N. Guberman et M. Soulières (2010). «Vivre et vieillir en milieu d'hébergement », dans M.Charpentier, N. Guberman, V. Billette, J.-P. Lavoie, A. Grenier et I. Olazabal (dir.), Vieillir au pluriel. Perspectives sociales, Québec, Presses de l'Université de Québec, p. 315-328).

Cloutier, E., David, H. et Teiger, C. (2003). « Agir sur les conditions de travail des auxiliaires de vie: croiser les approches ». Travail et emploi, $n^{\circ} 94$, p. 75-83.

Dooris, M. (2006). « Milieux de vie et promotion de la santé : orientations futures », Promotion \& Éducation, vol. 13, no 1, p. 16-18.Dooris, M. (2009). "Holistic and sustainable health improvment: the contribution of setting-based to approach to health promotion », Perspectives in Public Health, vol. 129, no 1, p. 29-36.

Dooris, M., B. Poland, L. Kolbe, E. Leeuw, D. McCall et J. Wharf-Higgins (2007). « Healthy Settings. Building evidence for the effectiveness for whole system promotion-Challenges and future directions », dans D. McQueen et C. Jones, Global Perspectives on Health Promotion Effectiveness, NewYork, Springer, p.327-352. 
Eynard, C., O. Piou et A. Villez(2009). Conduire le changement en gérontologie. Principes, méthodes et cas pratiques, Paris, Dunod.

Fahey, C. (2003). « Culture change in Long term care facilities: Changing the facility or changing the system? », Journal of Social Works in Long-Term Care, vol. 2, nos 1-2, p. 35-51.

Green, L.W., I. Rootman et B. Poland (1999). Settings for Health Promotion, Londres, Sage.

Guberman, N., et I. Olazabal (2010). « Vieillir où et avec qui ? Les environnements », dans M.Charpentier, N. Guberman, V. Billette, J.-P. Lavoie, A. Grenier et I. Olazabal (dir.), Vieillir au pluriel. Perspectives sociales, Québec, Presses de l'Université de Québec, p. 245-253.

Harris, N., J. Grootjans et K. Wenham (2009). « Ecological aging: the settings approach in aged living and care accommodation », EcoHealth, vol. 5, n², p. 196-204.

Harris, N., et J. Grootjans (2006). «The Potential Role of Ecological Health Promotion in Progressing Healthy Ageing », Ageing International, vol. 31, n²4, p. 276-282.

Houéto, D.,et G. Laverack (2014). Promotion de la santé et autonomisation dans le contexte africain, Raleigh (NC), Rossendale Books.

Kickbusch, I. (1996). « Tribute to Aaron Antonosky. "What creates health" », Health Promotion International, vol. 11, $\mathrm{n}^{\circ} 1$, p. 5-6.

Kickbusch, I. (2006). "La promotion de la santé comme un rhizome », dans M. O’Neill, S. Dupéré, A. P. Pederson et I. Rootman (dir.), Promotion de la santé au Québec et au Canada, perspectives critiques, Québec, Presses de l'Université Laval, p. 477-482.

Laforest, J. (1997). Gérontologie appliquée. Les professionnels de la vieillesse, Montréal, Hurtubise HMH Ltée.

Lalonde, M. (1974). Nouvelle perspective de la santé des Canadiens. Un document de travail, Ottawa, Gouvernement du Canada.

MSSS [Ministère de la Santé et des Services sociaux] (2003). Un milieu de vie de qualité pour les personnes âgées en CHSLD. Orientations ministérielles, Québec, Gouvernement du Québec.

MSSS [Ministère de la Santé et des Services sociaux] (2004). Un milieu de vie de qualité pour les personnes hébergées en CHSLD. Visites d'appréciation de la qualité des services, Québec, Direction des communications du ministère de la Santé et des Services sociaux.

MSSS [Ministère de la Santé et des Services sociaux] (2013). Rapport d'évaluation de la qualité de milieu de vie. Centre d'hébergement et soins de longue durée. Région Bas-SaintLaurent, Québec, Gouvernement du Québec.

Nutbeam, D. (1999). Glossaire de la promotion de la santé, Genève, Organisation mondiale de la santé.

OMS [Organisation mondiale de la santé] (1986). Charte d'Ottawa pour la promotion de la santé. Une conférence internationale pour la promotion de la santé, Ottawa, Organisation mondiale de la santé. 
O'Neill, M., A. Pederson, S. Dupéré et I. Rootman (2006). «La promotion de la santé au Canada et à l'étranger : bilan et perspectives », M. O'Neill, S. Dupéré, A.P. Pederson et I. Rootman (dir.), Promotion de la santé au Québec et au Canada, perspectives critiques, Québec, Presses de l'Université Laval, p. 3-21.

O'Neill, M., P. Simard, N. Sasseville, J. Mucha, B. Losier, B., L. McCue,D. McCall, M. Deschenes, D. Laitsch, F. Lagarde, T. Hancock, M.-C. Pelletier, M. Shain, A. Stirling et M. Niquette (2012). "Promoting Health trough the Settings Approach», dans I. Rootman, S. Dupéré, A, Pederson and M. O'Neill, Health Promotion in Canada. Critical perspectives on practice, Toronto, Canadian Scholar's Press Inc, p.171-192.

Ouellette, G. (2012). « Le travail en soins de longue durée : point de vue d'employés du centre d'hébergement du CSSS Cavendish », Pluriâges, vol. 3, nº 1, p. 6-9.

Pawson, R., et N. Tilley (1997). Realistic Evaluation, Londres et Thousand Oaks (CA), Sage.

Poland, B. Green L. et Rootman, I. (2000). Settings for Health Promotion: Linking Theory and Practice. Thousand Oaks/Newbury Park, CA: Sage Publications.

Poland, B., et M. Dooris (2010). «A green future and healthy future: the setting approach to building health, equity and susbtainability », Critical Public Health, vol. 20, n 3, p. 291-298.

Richard, L., L. Gauvinet K. Raine (2011). «Ecological models revisited. Their Uses and Evolution in Health Promotion over Two Decades », Public Health. Vol. 32, n 1, p.307-326.

Soulières, M., et G. Ouellette (2012). L'hébergement pour les personnes en perte d'autonomie au Québec. Des enjeux et des parcours difficiles pour les personnes concernées, Montréal, Regroupement provincial des comités des usagers.

Stokols, D. (1996). « Translating social ecological theory into guidelines for community health promotion », American Journal of Health Promotion, vol 10, $\mathrm{n}^{\circ} 4$, p. 282-98.

Villez, A. (2007). « EHPAD. La crise des modèles », Gérontologie et société, nº 123, p.169-184. 